\title{
ORDER PROPERTIES OF BOUNDED SELF-ADJOINT OPERATORS ${ }^{1}$
}

\author{
RICHARD V. KADISON
}

1. Introduction. It is the purpose of the present note to investigate the order properties of self-adjoint operators individually and with respect to containing operator algebras. We are particularly concerned with the vestiges of lattice structure which may remain in operator algebras when the commutativity assumption is dropped.

In brief the situation is as follows. ${ }^{2} \mathrm{~A}$ uniformly closed selfadjoint operator algebra ( $C^{*}$-algebra) $\mathfrak{A}^{\prime}$ which is commutative and contains the identity operator $I$ on the Hilbert space $\mathfrak{H C}$ is isomorphic in an algebraic and norm preserving fashion to a $C^{\prime}(X)$-see for example Stone $[3] .{ }^{3} C(X)$ (or for that matter $C(Y)$ when $I$ is not present in $\left.\mathfrak{A}^{\prime}\right)$ is a lattice, so that $\mathfrak{A}$ is a lattice in the ordering induced by the ordering on the (real) linear space $\widetilde{S}$ of all bounded self-adjoint operators on $\mathfrak{H C}^{4} \mathrm{~S}$. Sherman ${ }^{5}[2]$ has recently shown that if $\mathfrak{A}^{\prime}$ is a $C^{*}$-algebra with $\mathfrak{A}$ a lattice in the given order, then $\mathfrak{A}^{\prime}$ is commutative. Our results indicate that the more noncommutative $\mathfrak{A}^{\prime}$ becomes the less lattice structure $\mathfrak{A}$ retains. In fact our principal result states: $\subseteq$ is such that for $A, B$ in $\mathfrak{S}$, a greatest lower bound for $A$ and $B$ exists with respect to $\subseteq$ if and only if one of $A$ or $B$ is greater than or equal to the other ( $A$ and $B$ are comparable). A moment's thought shows that this is as strongly nonlattice as a partially ordered vector space can be. For this reason, we call such a system an anti-lattice. We shall also show that two projections in a $W^{*}$-algebra (weakly closed $C^{*}$-algebra) have a greatest lower bound

Presented to the Society, February 24, 1951; received by the editors April 21, 1950 and, in revised form, July $1,1950$.

${ }^{1}$ This paper was written while the author was an Atomic Energy Commission Predoctoral Research Fellow in the Physical Sciences of the National Research Council.

${ }^{2}$ We adopt the notation: $C^{\prime}(X)$ for the set of all complex-valued continuous functions on the compact Hausdorff space $X ; C(X)$ for the set of real functions in $C^{\prime}(X)$; $C^{\prime}(Y)$ for the set of all complex-valued continuous functions vanishing at $\infty$ on the locally compact Hausdorff space $Y ; C(Y)$ the set of real functions in $C^{\prime}(Y) . X$ will always denote a compact Hausdorff space and $Y$ a locally compact Hausdorff space.

${ }^{3}$ Numbers in brackets refer to the bibliography at the end of the paper.

4 The symbol for the algebra without the prime will be used to denote the real linear space of self-adjoint operators in the algebra.

- The author had access to the manuscript for S. Sherman's paper [2] in advance of publication. 
with respect to the algebra only if they commute (the converse is not true). We note later that if $\mathfrak{A}^{\prime}$ is a $C^{*}$-algebra such that $\mathfrak{A}$ is an anti-lattice, then $\mathfrak{A}^{\prime}$ is in a certain sense central simple.

\section{Commuting projections.}

THEOREM 1. Two projections commute if and only if they have a greatest lower bound with respect to some containing real linear space of self-adjoint operators which contains in addition the projection lattice join and intersection of the two given projections.

Leмма 2. If $E$ and $F$ are projections on the manifolds $M$ and $N$ respectively, then the projection $P$ on the manifold $M \cap N$ (that is, the projection lattice intersection of $E$ and $F$ ) is a greatest lower bound of $E$ and $F$ with respect to all positive self-adjoint operators.

Proof. Clearly $P \leqq E, F$; if $A \geqq 0$ and $A \leqq E, F$, then for $x$ in $M^{\perp}$ (the orthogonal complement of $M) 0=(E x, x) \geqq(A x, x) \geqq 0$ and thus $0=(A x, x)=\left(A^{1 / 2} x, A^{1 / 2} x\right)$, so that $A^{1 / 2} x=0$ and $A x=A^{1 / 2} A^{1 / 2} x=0$. Since $A$ annihilates $M^{\perp}$ and is self-adjoint, $A$ leaves $M$ invariant and thus $A \mathfrak{H C} \subset M$. By symmetry $A \mathfrak{H C} \subset N$ so that $A \mathfrak{H C} \subset M \cap N$. For arbitrary $z$ in $\mathcal{H}$ write $z=x+y$ with $x$ in $M \cap N$ and $y$ in $(M \cap N)^{\perp}$, then

$$
(P z, z)=(x, x)=(E x, x) \geqq(A x, x)=(A z, z) .
$$

LEMMA 3. If $a$ and $b$ are elements of a partially ordered vector space $V$ which have a positive greatest lower bound $c$ in $V$, then $a-c \leqq d$ for all positive $d$ such that $a \leqq b+d$.

Proof. Clearly $a-d \leqq a$, and from the hypothesis $a-d \leqq b$ thus $a-d \leqq c$ or $a-c \leqq d$.

Proof of Theorem 1 . Let $E^{\prime}$ and $F^{\prime}$ be the given projections, $\mathfrak{l}$ the real linear space, $G^{\prime}$ the greatest lower bound of $E^{\prime}$ and $F^{\prime}$ with respect to $\&, G$ and $P$ the projection lattice intersection and join of $E^{\prime}$ and $F^{\prime}$ respectively (all these operators in $\mathfrak{R}$ by hypothesis). Set $E$ equal to $E^{\prime}-G^{\prime}$ and $F$ equal to $F^{\prime}-G^{\prime}$. $E$ and $F$ have 0 as their greatest lower bound with respect to $\mathscr{R}$. In fact if $T \leqq E, F$, then $T+G^{\prime}$ $\leqq E^{\prime}, F^{\prime}$ so that, with $T$ in $\mathbb{R}, T+G^{\prime} \leqq G^{\prime}$ or $T \leqq 0$. $E^{\prime}$ and $F^{\prime}$ are both positive so that $G^{\prime}$ is positive, thus, by Lemma $2, G^{\prime} \leqq G$ so that $G=G^{\prime}$ and $E$ and $F$ are projections. Since $E$ and $F$ have 0 as a greatest lower bound, we have, by Lemma $3, E \leqq B$ for each positive operator $B$ in $\mathbb{R}$ such that $E \leqq F+B$. Choosing the projection $P-F$ for $B$, we see that $E \leqq F+P-F=P$ so that $E \leqq P-F$ and $E F=0$. Thus $E^{\prime}$ and $F^{\prime}$ commute.

If $E^{\prime}, F^{\prime}$ commute, clearly the $C^{*}$-algebra generated by $E^{\prime}, F^{\prime}$, $I$ is commutative and hence a lattice. 
CoROLlaRy 4. If two projections in a $W^{*}$-algebra $\mathfrak{A}^{\prime}$ have a greatest lower bound with respect to $\mathfrak{A}$ then they commute.

As a corollary of this result we get the following special case of Sherman's theorem [2].

COROLlaRY 5. A $W^{*}$-algebra $\mathfrak{A}^{\prime}$ such that $\mathfrak{A}$ is a lattice in the operator order is commutative.

Proof. If $\mathfrak{A}$ is a lattice, each pair of projections in $\mathfrak{X}^{\prime}$ commute by Corollary 4. Thus $\mathfrak{A}^{\prime}$ is commutative, since finite linear combinations of projections lie dense in $\mathfrak{A}^{\prime}$ (uniformly).

\section{Anti-lattices.}

TheOREM 6. $\subseteq$ is an anti-lattice.

Lemma 7. Two projections have a greatest lower bound with respect to $\mathbb{S}$ if and only if they are comparable.

Proof. Suppose $E^{\prime}$ and $F^{\prime}$ are projections with the greatest lower bound $G$ with respect to $\subseteq$. By Lemma $2, G$ is the projection lattice intersection of $E^{\prime}$ and $F^{\prime}$. As before we set $E$ equal to $E^{\prime}-G$ and $F$ equal to $F^{\prime}-G$ and observe that $E$ and $F$ have greatest lower bound 0 with respect to $\subseteq$. Thus, as in the proof of Theorem $1, E F=0$. If neither $E$ nor $F$ is 0 , there are nonzero vectors $x$ and $y$ such that $E x=x, F y=y$; so that $E y=0$ and $F x=0$. Let $M$ be the 2-dimensional space generated by $x$ and $y$ and $P$ the projection on it. Note that $E$ and $F$ leave $M$ invariant. $E$ and $F$ restricted to $M$ have the matrix forms

$$
\left(\begin{array}{ll}
1 & 0 \\
0 & 0
\end{array}\right) \text { and }\left(\begin{array}{ll}
0 & 0 \\
0 & 1
\end{array}\right)
$$

respectively relative to the basis $x, y$. The operator $A$ on $M$ whose matrix form is.

$$
\left(\begin{array}{cc}
-1 & 2^{1 / 2} \\
2^{1 / 2} & -1
\end{array}\right)
$$

relative to $x, y$ is less than both $E$ and $F$ on $M$ but not negative (the conditions for

$$
\left(\begin{array}{ll}
a & b \\
b & c
\end{array}\right)
$$

to be positive are $a, c \geqq 0$ and $a c \geqq b^{2}$ ). Thus the operator $A P$ on $\mathfrak{F C}$ 
is not negative. We claim $A P \leqq E, F$. In fact let $z$ be an arbitrary vector in $\mathcal{F}$ with $x^{\prime}$ its component in $M$ and $y^{\prime}$ its component in $\mathrm{M}^{\perp} ;$ then $(A P z, z)=\left(A x^{\prime}, x^{\prime}\right) \leqq\left(E x^{\prime}, x^{\prime}\right) \leqq(E z, z)=\left(E x^{\prime}, x^{\prime}\right)$ $+\left(E y^{\prime}, y^{\prime}\right)$, since $E$ leaves $M$ and consequently $M^{\perp}$ invariant. The same inequality holds for $F$. This contradicts the fact that $E$ and $F$ have 0 as a greatest lower bound with respect to $\subseteq$. Thus one of $E$ or $F$ is zero so that $E^{\prime}$ and $F^{\prime}$ are comparable.

Proof of Theorem 6. Suppose $A^{\prime}$ and $B^{\prime}$ have the greatest lower bound $C$ with respect to $\subseteq$. Let $A^{\prime}-C$ be $A$ and $B^{\prime}-C$ be $B$ : as before, $A$ and $B$ have the greatest lower bound 0 with respect to $\subseteq$. We complete the proof by showing that one of $A$ or $B$ is 0 . Suppose $A$ is nonzero. The $W^{*}$-algebra $\mathfrak{A}^{\prime}$ generated by $A$ and $I$ is representable as the set of all complex-valued continuous functions on a compact-Hausdorff space $X$ such that for $g$ a real continuous function on $X$, the closure of the set $[x: x$ in $X, g(x)>\delta]$ is a closed and open set. We denote by " $A$ " again the representing function for $A$. $A$ is a positive nonzero real function so that, for some small positive $\delta$, the set of points where $A$ is greater than $\delta$ is nonvoid. By the properties of $X$ the closure of this set is both closed and open, so that its characteristic function $E$ is continuous. Clearly $A \geqq \delta E$. Denoting by " $E$ " again the projection in $\mathfrak{A}^{\prime}$ corresponding to the function $E$, we have $A \geqq \delta E$ in $\mathfrak{A}^{\prime} . E$ and $B$ have the greatest lower bound 0 with respect to $\subseteq$. In fact if $T \leqq B$ and $T \leqq E$, then $\delta T \leqq \delta E \leqq A$ and $\delta T \leqq \delta B \leqq B$; thus $\delta T \leqq 0$ and $T \leqq 0$. By what we have proved, if $B$ were nonzero, we could find a nonzero projection $F$ such that $E$ and $F$ have 0 as a greatest lower bound with respect to $\subseteq$. But this is impossible for two nonzero projections, by Lemma 7 . Hence $B$ is 0 and the proof is complete.

COROLlARY 8. If $A$ and $B$ are self-adjoint operators in $a W^{*}$-algebra with greatest lower bound 0 , then $A B=0$. If $A$ and $B$ have a greatest lower bound $C$ which commutes with them, then $A$ and $B$ commute.

Proof. The last assertion follows from the first, for $A-C$ and $B-C$ have the greatest lower bound 0 so that $0=(A-C)(B-C)$ $=(B-C)(A-C)$ and $A B=C B+A C-C^{2}=B C+C A-C^{2}=B A$. In the case $A$ and $B$ have the greatest lower bound 0 , from the proof of Theorem 6, we see that each pair of projections $E$ and $F$ such that $A \geqq \delta E, B \geqq \gamma F, \delta, \gamma>0$ have greatest lower bound 0 with respect to the $W^{*}$-algebra. Thus, by Corollary $4, E$ and $F$ commute so that $E F=0$. But $A$ is a uniform limit of finite linear combinations of such projections $E$ (as is $B$ with $F$ ). Therefore $A B=0$.

The following theorem establishes more fully the relation between 
the anti-lattice character and the noncommutativity of an operator algebra.

Theorem 9. If the $C^{*}$-algebra $\mathfrak{A}^{\prime}$ is such that $\mathfrak{A}$ is an anti-lattice, then the center $\mathfrak{S}$ of $\mathfrak{A}^{\prime}$ is either (0) or the complexes.

LEMma 10. Each pair of commuting self-adjoint operators $A$ and $B$ have a greatest lower bound (and least upper bound) with respect to all self-adjoint operators which commute with them.

Proof. By considering the $C^{*}$-algebra containing $A, B$, and $I$ and its $C(X)$ representation, one finds that $C=(|A-B|+A+B) / 2$ is a least upper bound for $A$ and $B$ with respect to this algebra, where $|D|^{2}=D^{2}$ and $D \geqq 0$. If now $T \geqq A, B$ and $T$ commutes with $A$ and $B$, we have $T \geqq C$, for the $C^{*}$-algebra containing $T, A, B$, and $I$ contains $|A-B|$. Since the square root (positive) of a positive function is unique, the absolute value of $A-B$ is the same in both algebras, so that $C$ is the least upper bound with respect to the last algebra constructed and we have the desired conclusion.

Proof of Theorem 9. We suppose $\mathbb{E}$ is not (0). Being a commutative $C^{*}$-algebra, $\mathfrak{C}$ is representable as $C^{\prime}(Y)$. By Lemma 10 , each pair of self-adjoint operators in $\mathbb{E}$ has a greatest lower bound in $\mathfrak{A}$ with respect to $\mathfrak{A}$. Since $\mathfrak{A}$ is an anti-lattice, this last remark shows that the self-adjoint operators of $\mathfrak{E}$ are simply ordered. Thus the real functions in $C^{\prime}(Y)$ are simply ordered. This can occur only if $Y$ consists of a single point, and, thus, $\mathbb{E}$ is the complexes.

CoRoLlary 11. A $W^{*}$-algebra containing the identity operator which is an anti-lattice is a factor (see [1]).

A mild modification of the proof given for Theorem 6 enables us to complete the identification of lattice and commutativity properties. At least in the separable case, the results of Murray-von Neumann [1] show us that a factor $\mathfrak{M}$ of Type $I$ is algebraically isomorphic (and hence order isomorphic) to the set of all bounded operators on some (not necessarily infinite-dimensional) Hilbert space, so that such factors are anti-lattices. We shall now prove this result for arbitrary factors. A brief examination of [1] shows the comparability result we need $[1$, Lemma 6.2 .2$]$ to be valid for factors in the nonseparable case, so that our results are valid for general Hilbert space. In fact the comparability result needed involves only orthogonal projections and can be easily obtained in algebraic rather than spatial context.

Theorem 12. A factor $\mathfrak{M}$ is an anti-lattice. 


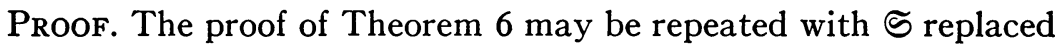
throughout by $\mathfrak{M}$ with the exception of Lemma 7 . To make the necessary replacement here, we are confronted with nonzero orthogonal projections $E$ and $F$ having 0 as alleged greatest lower bound with respect to $\mathfrak{M}$ : our task is to imitate the operator $A$ of Lemma 7 in the factor $\mathfrak{M}$. We can assume $E$ and $F$ are equivalent (in the sense of [1]); for either of $E$ and $F$ can be replaced by smaller nonzero projections in $\mathfrak{M}$ without changing the situation, and the comparability result quoted above completes the desired reduction.

Let $U$ be the partially isometric operator of $\mathfrak{M}$ establishing the equivalence between $E$ and $F$ ( $U$ carries the range $M$ of $E$ unitarily onto the range $N$ of $F$ and annihilates the null space of $E$ ). Let $A$ be $-E-F+2^{1 / 2}\left(U+U^{*}\right)$. We assert $E-A=2 E+F-2^{1 / 2}\left(U+U^{*}\right) \geqq 0$ (and symmetrically $F-A \geqq 0$ ). In fact, for arbitrary $w$ in $\mathcal{H C}$ we write $w=x+y+z$ with $x$ in $M, y$ in $N$, and $z$ in $\mathcal{H} \ominus(M \oplus N)$. Then $((E-A) w, w)=2(x, x)+(y, y)-2^{1 / 2}\left((U x, y)+\left(U^{*} y, x\right)\right)=2\|x\|^{2}$ $+\|y\|^{2}-2\left(2^{1 / 2}\right) R(U x, y)$. But $R(U x, y) \leqq|(U x, y)| \leqq\|U x\|\|y\|=\|x\|$ $\|y\|$, so that $((E-A) w, w) \geqq 2\|x\|^{2}-2\left(2^{1 / 2}\right)\|x\|\|y\|+\|y\|^{2}$. Now $2\|x\|^{2}$ $-2\left(2^{1 / 2}\right)\|x\|\|y\|+\|y\|^{2} \geqq 0$ since

$$
\left(\begin{array}{cc}
2 & -2^{1 / 2} \\
-2^{1 / 2} & 1
\end{array}\right)
$$

is positive definite (as noted in Lemma 7), and thus $E-A \geqq 0$ as asserted. We also have $0 \ngtr A \neq 0$, for with $x \neq 0$ in $M, U x$ is in $N$ so that $(A(x+U x), x+U x)=-(x, x)+2^{1 / 2}(U x, U x)+2^{1 / 2}(x, x)-(U x, U x)$ $=2\left(2^{1 / 2}-1\right)(x, x)>0$. It follows that $E$ and $F$ do not have the greatest lower bound 0 in $\mathfrak{M}$ and the proof is complete.

Theorem 12 in conjunction with Corollary 11 single out for us among the class of all rings of operators those which are anti-lattices: they are the factors.

\section{BiBLIOGRAPHY}

1. F. Murray and J. von Neumann, On rings of operators, Ann. of Math. vol. 37 (1936) pp. 116-229.

2. S. Sherman, Order in operator algebras, Amer. J. Math. vol. 73 (1951) pp. 227232.

3. M. H. Stone, $A$ general theory of spectra I, Proc. Nat. Acad. Sci. U.S.A. vol. 26 (1940) pp. 280-283.

University of Chicago 